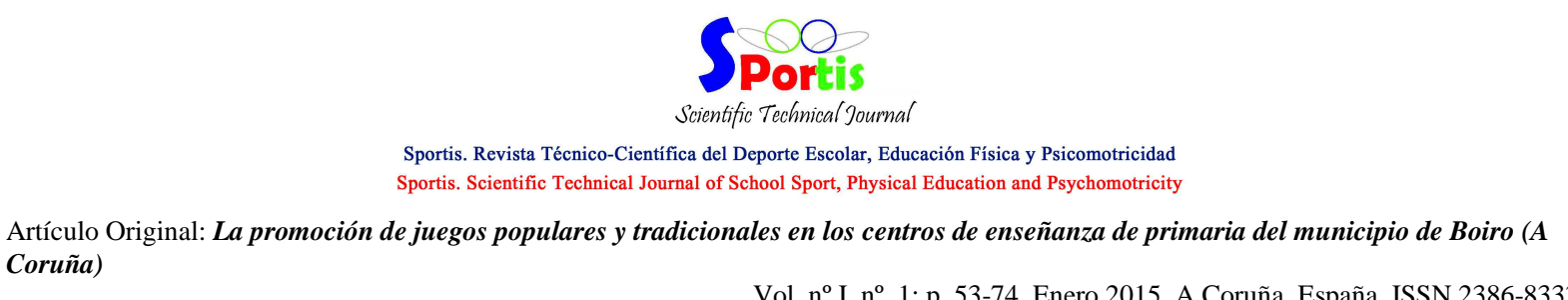

Vol. $n^{\circ}$ I, n ${ }^{\circ} .1 ;$ p..53-74, Enero 2015. A Coruña. España ISSN 2386-8333

\title{
La promoción de juegos populares y tradicionales en los centros de enseñanza primaria del municipio de Boiro (A Coruña)
}

\section{The promotion of popular and traditional games in elementary education of Boiro's municipality (A Coruña)}

\author{
Rodríguez Fernández, J.E ${ }^{1}$; Pazos Couto, J.M².; Palacios Aguilar, J. ${ }^{3}$ \\ ${ }^{1}$ Universidade de Santiago de Compostela; ${ }^{2}$ Universidade de Vigo; ${ }^{3}$ Universidade da Coruña
}

Contacto: uxiorf@hotmail.com

Cronograma editorial: Artículo recibido: 24/12/2014 Aceptado: 07/01/2015 Publicado: 08/01/2015

\section{Resumen}

El actual currículum para la Educación Primaria en la Comunidad Autónoma de Galicia recoge la importancia de los juegos populares y tradicionales gallegos y establece los contenidos a trabajar en toda esta etapa educativa, basados principalmente en el reconocimiento, práctica, investigación, historia y valor cultural que este tipo de prácticas tiene para el alumnado de esta comunidad. El juego de bolos en Boiro tuvo en otros tiempos una gran repercusión entre la ciudadanía, siendo probablemente el principal divertimiento de los boirenses en el primer tercio del siglo XX. El objetivo de este estudio es analizar la promoción, difusión y práctica de los juegos populares y tradicionales en general y del juego de bolos en particular en los centros de enseñanza primaria del municipio de Boiro, utilizando técnicas cualitativas para la obtención de datos, siendo la entrevista personal la principal herramienta utilizada para tal fin. Los resultados del estudio nos muestran la necesidad de profundizar en el trabajo de estas prácticas lúdicas en los centros de enseñanza, hoy en día más orientados a cumplir con lo mínimo establecido en el currículum oficial que en dotar de verdadero significado a juegos que forman parte de la historia y cultura del pueblo de Boiro, siendo el juego de bolos la referencia más clara en este sentido. Las Administraciones Públicas deben coordinarse para poner en práctica un proyecto mucho más ambicioso de recuperación de este tipo de prácticas, en el que la escuela debe considerarse como el punto de partida. 


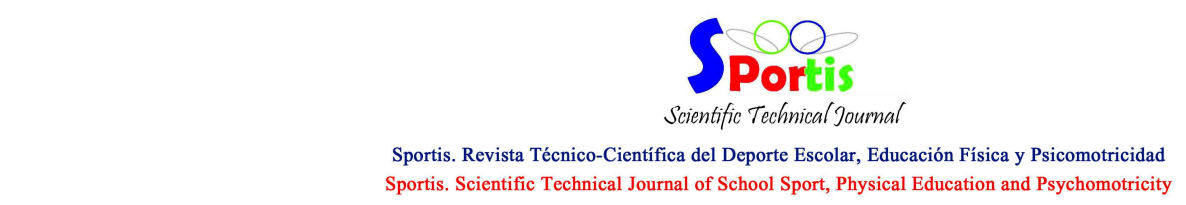

Artículo Original: La promoción de juegos populares y tradicionales en los centros de enseñanza de primaria del municipio de Boiro (A Coruña)

Vol. $n^{\circ}$ I, $n^{\circ}$. 1; p..53-74, Enero 2015. A Coruña. España ISSN 2386-8333

\section{Abstract}

The current curriculum for elementary education in the autonomous community of Galicia includes the importance of Galician traditional and popular games and sets the content to work on all this educational stage, mainly based on recognition, practice, research, history and cultural value that this type of practice has for the students of this community. The skittles's game in Boiro in the past had a great repercussion among the people, being probably the principal entertainment of the Boiro's people in the first third of the 20th century. The objective of this study is to analyze the promotion, dissemination and practice of popular and traditional games in general and the skittles's game in particular into the elementary schools of the municipality of Boiro, using qualitative techniques for data collection, the personal interview to be the main tool used for this purpose. The results of the study show the need to deepen the work of these recreational practices in education centers, nowadays more oriented to comply with the minimum laid down in the official curriculum that give true meaning to games that are part of the history and culture of the village of Boiro, still skittles's game clearer reference in this regard. Public administrations must be coordinated to implement a much more ambitious project of recovery of this kind of practice, in which the school should be considered as the starting point.

Palabras clave: educación primaria, Edúcación Física, juegos populares y tradicionales, bolos, Boiro.

Key words: elementary education, Physical Education, popular and traditional games, skittles, Boiro.

\section{Introducción}

El juego, como una actividad necesaria para los seres humanos (Veiga, 1998), ha estado siempre presente a lo largo de la historia de la humanidad, existiendo numerosas pruebas provenientes de estudios de las culturas antiguas.

Como herramienta útil para adquirir y desarrollar actividades intelectuales, motoras o afectivas (Bautista, 2002; Melero, 2009), requiere ser realizado de forma voluntaria y placentera, sin sentir obligación de ningún tipo y disponiendo del tiempo y espacio necesarios para extraer la verdadera esencia de esta actividad libre, espontánea, voluntaria, innata y que, 
además, como afirman autores como García y Alarcón (2011), Renzi (2009) o Ruiz (2011), es una actividad muy relacionada con las primeras etapas de la vida, con la infancia.

A edades tempranas, el juego está presente en todos los momentos de la vida del niño, formando parte de su repertorio de actividades que le proporcionarán numerosos beneficios (Barroso, 2003; Lavega 2006), ya que además de cumplir con la teoría del excedente de energía (Monroy y Sáez, 2011), podrá adquirir notables mejoras a nivel psicomotor, cognitivo, social y afectivo (Lara, 2011; Rodríguez, Pazos y Palacios, 2014; Romero 2007).

Todas estas cualidades positivas se mostrarán principalmente en los dos ambientes más importantes en los que el niño invierte su tiempo: en contacto con la familia y durante su etapa educativa en la escuela (Ponce, 2009).

El juego, como recurso educativo y escolar, ha de llevar implícito el desarrollo de aspectos emocionales, afectivos, creativos y componentes que fomenten su autonomía personal (Ponce, 2009). El desarrollo de todas estas capacidades en el ámbito escolar requiere de la implicación de todo el profesorado, sobre todo del especialista en Educación Física, que a su vez necesita que las Administraciones Educativas recojan en los documentos curriculares que elaboren la forma de incrementar y potenciar este tipo de actividades que emplean el juego como medio o como fin en si mismo.

En el actual currículum de Educación Primaria para la Comunidad Autónoma de Galicia (DOG 171, de 9 de septiembre de 2014, p. 37785) se recoge que "la Educación Física a lo largo de la educación primaria tiene en el juego el más importante recurso como situación de aprendizaje y herramienta didáctica por su carácter motivador. Se debe destacar los juegos tradicionales gallegos que, junto con las danzas y bailes propios de Galicia, suponen un elemento importante de transmisión de nuestro patrimonio artístico y cultural, además de fomentar las relaciones intergeneracionales"

Bajo la premisa de que la Educación Física en esta etapa tendrá un carácter eminentemente global y lúdico, hasta el punto de convertir el juego en el contexto ideal para la mayoría de los aprendizajes, se recoge en el bloque 6 de contenidos (los juegos y actividades deportivas) la forma de tratar este recurso educativo a lo largo de toda la etapa, siendo el reconocimiento, práctica, investigación, riqueza cultural y valor de los juegos y deportes populares gallegos una parte importante de este bloque de contenidos que, evidentemente, es de obligado cumplimento por parte de los docentes del área de Educación Física. 
Galicia, al igual que el resto de las Comunidades Autónomas del territorio español, posee un importante bagaje de juegos populares y tradicionales, determinados por las costumbres de la gente (Vega, 1990), los trabajos propios de la zona (Veiga, 1998), la zona de poblamiento (Cortizas, 2001; Romaní, 1979) o incluso el sexo del participante (Liñares, 2007). Estos juegos, transmitidos de generación en generación (Moreno, 1992), constituyen el reflejo, la identidad y cultura de un pueblo (Lavega, 1994; Vigne, 2011); y Galicia, a pesar de no ser una comunidad que destaque precisamente por conservar sus tradiciones, sigue conservando la práctica del juego de bolos, uno de los juegos populares y tradicionales más importantes del mundo por su antigüedad, universalidad y presencia en todo el mundo (Expósito, 2006; Lavega, 1996; López, 2009; Ruiz, 2000).

La práctica del juego de bolos en Galicia es diferente en las cuatro provincias, en cuanto a modalidad, cantidad e intensidad de práctica, siendo A Coruña la que menos actividad registra. De hecho, sólo está constatada actividad bolística en el municipio de Boiro localidad que posee una historia muy importante en relación a este juego milenario (Rodríguez, 2013).

El objetivo de esta investigación es analizar la práctica de los juegos populares y tradicionales en general, y del juego de bolos en particular, en los centros de educación primaria del municipio de Boiro (A Coruña).Su tratamiento en el área de Educación Física, la importancia de la escuela en la promoción de estas actividades y el análisis de las causas que motivaron su paulatino descenso en el nivel de práctica, son variables en las que se profundizará en este estudio.

\section{Material y método}

\section{Localización}

El estudio se llevó a cabo en Boiro, municipio situado al oeste de la provincia de A Coruña, en pleno corazón de las Rías Bajas gallegas. De tamaño mediano (casi 19.200 habitantes), dispone en activo de 2 escuelas de Educación Infantil (dependientes de un CEIP), 6 centros de Educación Infantil y Primaria y 3 institutos de Educación Secundaria, que en total escolarizan un total de 2.496 alumnos.

\section{Temporalización}

La toma de datos fue realizada a finales del año 2012. Este trabajo proviene además de la tesis doctoral leída en la Universidad de A Coruña en el año 2013: “O estudo do xogo dos 
bolos en Boiro: aspectos históricos, estado actual de práctica e perspectivas de futuro", elaborada por el principal autor de este artículo.

\section{Diseño, participantes e instrumento de recogida de datos}

Se emplea una metodología cualitativa (Canales, 2006; Tójar, 2006) de corte descriptivo (Corbetta, 2007), con el objetivo de profundizar en las opiniones de las personas que participan en el estudio. La muestra estuvo formada por los 8 profesores del área de Educación Física de los 6 Centros de Educación Infantil y Primaria (7 hombres y 1 mujer).

Se utilizó la entrevista personal como herramienta metodológica para la obtención de datos (Canales 2006; Tójar 2006). Se trata de una entrevista semiestructurada orientada a indagar en el trabajo de aula del profesional de la Educación Física y del papel de la escuela en la promoción y desarrollo de los juegos populares y tradicionales.

En este caso, constituye una herramienta de gran utilidad por el conocimiento personal previo de cada uno de los entrevistados, que empleada con flexibilidad y en un clima confortable, permitió obtener datos con un mayor grado de fiabilidad y exhaustividad.

\section{Procedimiento}

Dadas las características tan concretas de la muestra, se establecen las entrevistas personales en el centro propio de cada maestro. Grabadas en audio y video, se desarrolla la entrevista y posteriormente se procede a la transcripción de las mismas.

Una vez descargados los datos, se realiza el tratamiento de la información recogida, donde el objetivo principal fue realizar una reducción cualitativa de datos (Tójar, 2006) con el fin de hacer más manejable e interpretable la información. Se procede a identificar las unidades de información en el conjunto de las entrevistas, se agruparon por temas y, posteriormente, se definieron las categorías, tal y como queda reflejado en la figura 1.

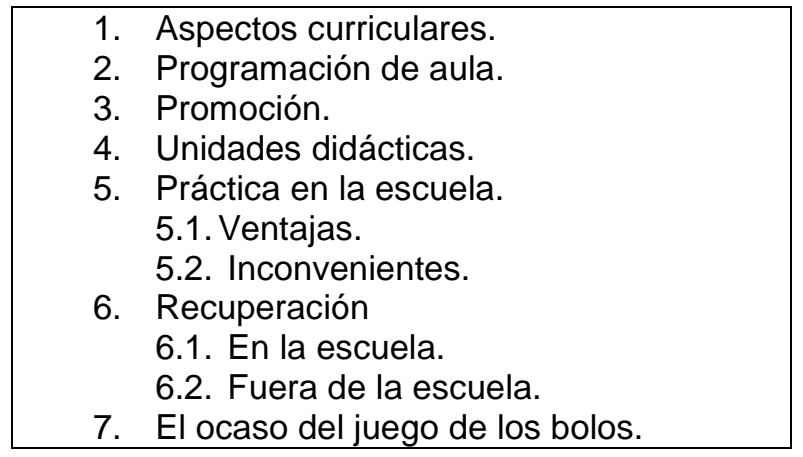

Figura 1. Categorías objeto de estudio. 
Artículo Original: La promoción de juegos populares y tradicionales en los centros de enseñanza de primaria del municipio de Boiro (A Coruña)

\section{Resultados y discusión}

En este apartado se analiza de qué forma se promueven los juegos populares y tradicionales y, entre ellos, el juego de bolos, en los centros educativos del Ayuntamiento de Boiro.

En la categoría 1 (maestros-importancia de los juegos populares y tradicionales en el currículum de Educación física) (ver tabla 1) destacan las respuestas $(62,5 \%)$ que indican que los juegos populares y tradicionales están presentes en el currículum de Educación Primaria (p.e. maestro 4: los juegos populares y tradicionales aparecen dentro del currículum tanto en el objetivo de área como en los contenidos y en los criterios de evaluación en primero, segundo y tercer ciclo. No aparecen así, o no tengo constancia, en Educación Infantil).

En contraposición a esto, aparecen respuestas (12,5\%) que indican que los juegos populares y tradicionales tienen poca presencia en el currículum (p.e. maestro 7: lo que es desde el currículum de Educación Física, poco).

Aparecen también respuestas $(12,5 \%)$ que hacen referencia a que se amplió su presencia en el currículum de Educación Física (p.e. maestro 1: que es verdad que la Consellería de Educación intentó, amplió, porque antes no había nada de juegos populares y tradicionales; dentro del currículum no era ni un bloque de contenidos, era un punto y bien, ahora lo ampliaron más) y respuestas $(12,5 \%)$ que dicen que constituye un bloque importante dentro del currículum (p.e. maestro 3: a mi me parece que los juegos populares y tradicionales son un bloque importante en el currículum de Educación Física).

\begin{tabular}{lc}
\hline \multicolumn{1}{c}{ Respuesta/s } & $\begin{array}{c}\text { №. de menciones en las } \\
\text { entrevistas }\end{array}$ \\
\hline Presencia en el currículum de Educación Física & 5 \\
\hline $\begin{array}{l}\text { Se amplió su presencia en el currículum de Educación } \\
\text { Física }\end{array}$ & 1 \\
\hline Constituye un bloque importante en el currículum & 1 \\
\hline Tienen poca presencia en el currículum & 1 \\
\hline Tabla 1. Categoría 1. Maestros-importancia de los juegos populares y tradicionales en el currículum \\
$\quad$ de Educación Física
\end{tabular}

Pensamos que en este punto es preciso matizar una serie de cuestiones. Por un lado, está el antiguo Decreto 130/2007, de 28 de junio, por el que se establece el currículum de Educación 
Primaria en la Comunidad Autónoma de Galicia, vigente en el momento de realizar las entrevistas. En este documento vienen reflejados, entre otros, los objetivos, competencias básicas, contenidos, criterios de evaluación y orientaciones metodológicas para las distintas áreas que constituyen la etapa de primaria, entre ellas, el área de Educación Física. En este decreto, se reflejaban los contenidos a tratar en $1^{\circ}, 2^{\circ}$ y $3^{\circ}$ ciclo (bloque 5 de juegos y deportes) y los correspondientes criterios de evaluación. Con este documento de referencia, no entendemos como la totalidad de los maestros no responde que los juegos populares y tradicionales están dentro del currículum oficial en el área de Educación Física para la etapa de Educación Primaria y que constituyen un apartado importante dentro del bloque de juegos y deportes. A partir de aquí, los maestros tienen libertad para programar, por lo que podrán incidir en mayor o menor medida en alguno de los aspectos del currículum y dotar de mayor o menor importancia aquellos contenidos que consideren más relevantes. Creemos que los juegos populares y tradicionales tienen un peso importante dentro del actual currículum de Educación Física en primaria, estableciendo orientaciones claras para su conocimiento y práctica, insistiendo que es un deber que en la escuela se trabajen los juegos tradicionales gallegos y se enseñe al alumnado a jugar como se hacía antiguamente, reforzando de este modo al vínculo de niños y niñas con las familias (madres, padres, abuelos o abuelas).

En la categoría 2 (maestros-importancialde los juegos/populares y tradicionales en la programación de aula) (ver tabla 2) destacan las respuestas (50\%) que ponen especial énfasis en la importancia de los juegos populares y tradicionales en la programación de aula de los maestros (p.e. maestro 1: bien, yo le doy bastante importancia, porque en todos los trimestres intento tocar algo de juegos populares). A partir de aquí nos encontramos con dos tipos de respuestas que hacen referencia al tratamiento de los juegos populares y tradicionales durante el curso escolar. Así, el 37,5\% indican que emplean en sus sesiones de Educación Física los juegos populares y tradicionales a lo largo de todo el curso (p.e. maestro 6: y están en todos los trimestres, casi todos los meses tienen algo de juego popular), mientras que un porcentaje similar $(37,5 \%)$ dicen que sólo trabajan los juegos populares y tradicionales de forma puntual, en algún momento muy concreto del año (p.e. maestro 3: yo tengo para cada ciclo una unidad didáctica de juegos populares y tradicionales). En menor medida aparecen respuestas (25\%) que indican que sólo se emplea el juego popular y tradicional como medio para conseguir otros objetivos más importantes (p.e. maestro 2: yo tengo una forma de llevar las 


\begin{tabular}{|c|c|}
\hline \multicolumn{2}{|c|}{$\begin{array}{l}\text { Sportis. Revista Técnico-Cientifica del Deporte Escolar, Educación Física y Psicomotricidad } \\
\text { Sportis. Scientific Technical Journal of School Sport, Physical Education and Psychomotricity }\end{array}$} \\
\hline \multirow{3}{*}{\multicolumn{2}{|c|}{$\begin{array}{l}\text { Artículo Original: La promoción de juegos populares y tradicionales en los centros de enseñanza de primaria del municipio de Boiro ( } \\
\text { Coruña) } \\
\text { vol. } \mathrm{n}^{\circ} \mathrm{I}, \mathrm{n}^{\circ} .1 ; \text { p..53-74, Enero 2015. A Coruña. España ISSN } 2386- \\
\text { sesiones de Educación Física prácticamente a través de los juegos, y dentro de eses jue } \\
\text { muchas veces ya se están realizando juegos populares). }\end{array}$}} \\
\hline & \\
\hline & \\
\hline Respuesta/s & $\begin{array}{c}\text { №. de menciones en las } \\
\text { entrevistas }\end{array}$ \\
\hline Le doy mucha importancia & 4 \\
\hline Trabajo los juegos populares a lo largo del curso & 3 \\
\hline Trabajo los juegos populares de forma puntual & 3 \\
\hline Empleo los juegos populares como medio & 2 \\
\hline Su importancia es competencia de todos & 1 \\
\hline
\end{tabular}

Otras respuestas $(12,5 \%)$ que señalan que la importancia de los juegos populares y tradicionales y de su empleo en los centros educativos es competencia de todos, desde las diferentes administraciones públicas hasta el propio maestro de Educación Física (p.e. maestro 7: es competencia de todas las administraciones, no cabe duda, es nuestra cultura, es nuestro patrimonio, y tenemos que protegerlo, y a quien le importa es a todos los que estamos implicados, desde la Xunta de Galicia hasta los ayuntamientos y hasta la comunidad escolar, los padres, maestros y, en general, toda la sociedad). Entendemos que la importancia de los maestros le otorgan a los juegos populares y tradicionales debe traducirse en una mayor presencia en la práctica de éstos en sus clases de Educación Física. Pero vemos como a pesar de que indican que les dan mucha importancia a los juegos populares y tradicionales en sus programaciones de aula, suelen emplearlos como medio para conseguir otros objetivos, de forma puntual o para completar la programación en días en los que el centro celebra algún tipo de acto: Letras Galegas, magosto o final de curso. Creemos que los profesores de los centros de Educación Primaria de la localidad deberían conocer las tradiciones culturales y deportivas que afectan directamente a su alumnado, las que tuvieron o pudieron tener cierta relevancia en el municipio y, a partir de ahí, dotar de contenido concreto y específico a esa actividad, dándole la importancia que realmente tiene.

\footnotetext{
Para citar este artículo utilice la siguiente referencia: Rodríguez, J.E.; Pazos, J.M.; Palacios, J.. (2015). La promoción de juegos populares y tradicionales en los centros de enseñanza de primaria del municipio de Boiro (A Coruña). Sportis Scientific Technical Journal, 1 (1), 53-74 www.sportis.es 
Artículo Original: La promoción de juegos populares y tradicionales en los centros de enseñanza de primaria del municipio de Boiro (A Coruña)

Vol. $n^{\circ}$ I, $n^{\circ} .1 ;$ p..53-74, Enero 2015. A Coruña. España ISSN 2386-8333

Por otro lado, entendemos también que la carga horaria de la asignatura de Educación Física (dos horas a la semana) no es precisamente amplia como para tratar en profundidad todos los aspectos que se recogen en el bloque de contenidos del currículum.

Quizás antes de pedirle a los maestros que trabajen en profundidad un aspecto (como puede ser la práctica de un juego popular y tradicional en concreto), habría que proporcionarle las condiciones adecuadas para poder realizarlo y, entre otras, la carga horaria semanal de la asignatura de Educación Física en esta etapa debería ampliarse notablemente.

En la categoría 3 (maestros-promoción de los juegos populares y tradicionales en los centros educativos) (ver tabla 3) destacan respuestas (62,5\%) que indican que en los centros de primaria de Boiro se organizan actividades de juegos populares y tradicionales en días especiales del calendario (día de las Letras Galegas y carnaval preferentemente) (p.e. maestro 1: día del magosto, ese día, además de asar castañas, pues celebramos y hacemos juegos populares; el día de las Letras Galegas, final de curso, sobre todo).

\begin{tabular}{lc}
\hline \multicolumn{1}{c}{ Respuesta/s } & $\begin{array}{c}\text { №. de menciones en las } \\
\text { entrevistas }\end{array}$ \\
\hline $\begin{array}{l}\text { Se organizan actividades en días especiales } \\
\text { Se organizan actividades periódicamente en los } \\
\text { recreos }\end{array}$ & 3 \\
\hline $\begin{array}{l}\text { Se organizan los espacios para la práctica de los } \\
\text { juegos populares y tradicionales }\end{array}$ & 1 \\
\hline $\begin{array}{l}\text { Se organizan actividades en colaboración con las } \\
\text { ANPAS }\end{array}$ & 1 \\
\hline $\begin{array}{l}\text { Tabla 3. Categoría 3. Maestros-promoción de los juegos populares y tradicionales en los centros } \\
\text { educativos. }\end{array}$
\end{tabular}

En menor medida se muestran respuestas $(37,5 \%)$ que informan de que el período de tiempo que se emplea para la promoción de los juegos populares y tradicionales en los centros de enseñanza primaria es el que corresponde al recreo diario, realizándose de forma periódica y habitual (p.e. maestro 2: los viernes los tenemos dedicado, y así está reflejado en la Programación General Anual, que ese día se realizarán juegos populares y tradicionales). Y un bajo porcentaje de respuestas $(12,5 \%)$ subrayan que se promueven los juegos populares y tradicionales en el centro organizando espacios concretos para la realización de estos juegos (p.e. maestro 2: pues a la hora de la organización de espacios, por ejemplo, en la sesión del recreo, tenemos los espacios repartidos por ciclos, para que cada ciclo, de forma rotatoria, 
Artículo Original: La promoción de juegos populares y tradicionales en los centros de enseñanza de primaria del municipio de Boiro (A Coruña)

Vol. $n^{\circ}$ I, $n^{\circ} .1 ;$ p..53-74, Enero 2015. A Coruña. España ISSN 2386-8333 pueda utilizarlos) y organizando actividades en colaboración con las ANPAS (p.e. maestro 4: por parte del ANPA digamos que también tenemos mucha colaboración, y no hace tanto pintaron en el patio el juego de la mariola, y pintaron también el de los bolos). En referencia a la anterior categoría, observamos como los juegos populares y tradicionales se emplean más para completar la programación en días señalados del calendario que por el contenido y fin que deberían tener en si mismos. Pensamos que en este caso se intenta más cumplir con el mínimo exigido en el currículum que en dotar de verdadero significado este tipo de juegos, restándole el protagonismo e importancia que realmente tienen. Está bien que en estos días de fiesta se practiquen juegos populares y tradicionales, pero esto debería ser parte de un proyecto mucho más amplio de conocimiento, búsqueda, recopilación de información y práctica de esta tipología de actividades, primando los propios de la localidad o zona en la que se encuentra el centro educativo en cuestión. En la categoría 4 (maestros-los bolos en las unidades didácticas de juegos populares y tradicionales) (ver tabla 4) la totalidad de las respuestas (100\%) señalan que el juego de bolos puede formar parte de las unidades didácticas que los maestros elaboran conforme a sus programaciones (p.e. maestro 2: si, claro que pueden formar parte, pero no tendría porque ser una unidad didáctica específica). En la mayoría de los casos $(37,5 \%)$ que se promueve el juego de bolos en las clases de Educación Física, se emplean más como medio para conseguir otros objetivos que como fin en si mismos (p.e. maestro 4: digamos que son juegos de punterías en los que trabajamos la percepción de distancias y demás...). 


\begin{tabular}{|c|c|}
\hline Respuesta/s & $\begin{array}{l}\text { №. de menciones en las } \\
\text { entrevistas }\end{array}$ \\
\hline $\begin{array}{l}\text { Los bolos pueden formar parte de las unidades } \\
\text { didácticas }\end{array}$ & 8 \\
\hline $\begin{array}{l}\text { Se emplean como medio para conseguir otros } \\
\text { objetivos }\end{array}$ & 3 \\
\hline $\begin{array}{l}\text { Es difícil dedicar una unidad didáctica específica a los } \\
\text { bolos }\end{array}$ & 1 \\
\hline Es un juego peligroso & 1 \\
\hline No es un juego peligroso & 1 \\
\hline Se promueve una modalidad muy sencilla & 1 \\
\hline $\begin{array}{l}\text { Se necesita material adecuado para realizar la } \\
\text { actividad en el centro }\end{array}$ & 1 \\
\hline
\end{tabular}

\section{tradicionales.}

En menor medida, nos encontramos con respuestas (12,5\%) que dicen que es difícil dedicar una unidad didáctica específica de bolos en sus clases, sobre todo, por falta de tiempo para tratar todo el programa establecido y tocar otro tipo de juegos y deportes (p.e. maestro 5: lo que si, una unidad didáctica única y exclusivamente de bolos me parecería un poco exagerado), y respuestas $(12,5 \%)$ que muestran sus quejas de que no se dispone de material adecuado para realizar este tipo de actividades en el centro educativo (p.e. maestro 8: en este colegio concretamente, este es el segundo año, no lo tuve tan establecido porque no dispongo de material y entonces lo que es el juego de bolos, tanto el bolo celta como el resto de bolos, no los tengo incluidos correctamente). Otras respuestas (12,5\%) indican que lo que realmente desarrollan en el centro es una modalidad de bolos muy sencilla, refiriéndose concretamente a modalidades de derribo, escapando un poco de la esencia del juego de bolos de Boiro, una modalidad en la que lo importante es desplazar los bolos lo más lejos posible (p.e. maestro 3: los bolos que jugamos aquí en el colegio son unos bolos muy sencillos, pienso que no son los tradicionales de aquí de la zona). Y, por último, nos encontramos con respuestas contradictorias, ya que unas $(12,5 \%)$ afirman que el juego de bolos no es peligroso y que se puede realizar perfectamente en el centro educativo (p.e. maestro 1: no es peligroso, bien, peligroso no es ninguno, pero siempre andas con más cuidado) y otras $(12,5 \%)$ que afirman 
lo contrario, que el juego de bolos es un juego peligroso, debido al daño que puede producir el impacto de la bola o de los bolos en los propios participantes (p.e. maestro 6: pero el juego así, dentro del colegio, yo no lo metería, simplemente por el grado de peligrosidad que le veo al juego en si). La mayoría de los maestros de los centros de educación primaria de Boiro desconocen por completo el juego de bolos tradicionales característico de la localidad, por lo que entendemos que no le den la importancia que merece. Quizás el trabajo previo debe salir del Servicio Municipal de Deportes del Ayuntamiento de Boiro, informando a los maestros especialistas en Educación Física (y también al resto de la comunidad escolar) de la realidad del juego, de la importancia que tuvo en otros tiempos e implicándolos en un atractivo proyecto de recuperación y promoción de esta modalidad en todo el municipio. De este modo, pensamos que los maestros podrían dedicar unidades didácticas específicas al juego de bolos característico de Boiro, dotándolo de contenido y con un fin en si mismo, que es la historia, el conocimiento y la práctica en las clases de Educación Física de este juego popular y tradicional de un modo continuado a lo largo del curso escolar, potenciándolo aún más en fechas significativas, y aprovechando para que los niños y niñas les muestren a sus padres y familiares sus destrezas en una actividad que, seguramente, conocen perfectamente.

En la categoría 5 (maestros-ventajas de la práctica del juego de bolos en la escuela) (ver tabla 5) destacan las respuestas (100\%) que ven en el juego de bolos un buen medio para adquirir nuevas habilidades y de relación social (p.e. maestro 4: pues a nivel físico eso, adquirir nuevas habilidades, adquirir apreciación de espacios, distancias, punterías y también tenemos la parte de la relación con la familia, con los abuelos...).

En menor grado, se muestran respuestas (25\%) que destacan como una ventaja en la aplicación del juego de bolos en los centros de enseñanza la de tratarse de un juego que no es peligroso (p.e. maestro 1: no es peligroso, bien, peligroso no es ninguno, pero siempre andas con más cuidado) y respuestas $(12,5 \%)$ que indican que las ventajas residen en su naturaleza novedosa e interesante para los alumnos y alumnas (p.e. maestro 3: a mi me parece interesante; pienso que así los niños conocerían este tipo de juegos y jugarían más a los bolos). 


\begin{tabular}{lc}
\hline \multicolumn{1}{c}{ Respuesta/s } & $\begin{array}{c}\text { №. de menciones en las } \\
\text { entrevistas }\end{array}$ \\
\hline $\begin{array}{l}\text { Es un buen medio para adquirir nuevas habilidades y } \\
\text { de relación social }\end{array}$ & 8 \\
\hline No es un juego peligroso & 2 \\
\hline Es una actividad interesante y novedosa & 1 \\
\hline \multicolumn{1}{c}{ Tabla 5. Categoría 5. Maestros-ventajas de la práctica del juego de bolos en la escuela }
\end{tabular}

Coincidimos con los maestros en las ventajas que tiene la práctica del juego de bolos en la escuela, sobre todo en que es un medio estupendo para adquirir nuevas habilidades motrices y de relación social. Pero pensamos que las verdaderas ventajas que tendría la práctica del juego de bolos en los centros educativos de la localidad habría que buscarlas en la esencia del propio juego, en el aspecto de recuperar una tradición importante en el pueblo, en establecer continuos retos con uno mismo y con los demás, en el respeto, en la colaboración y en la satisfacción que produce ser la persona que continúa con una tradición que seguramente iniciaron sus padres, abuelos, tatarabuelos o quizás generaciones anteriores.

Resaltar de la categoría 6 (maestros-inconvenientes de la práctica del juego de bolos en la escuela) (ver tabla 6) que el 62,5\%) de las respuestas indican que no le encuentran ningún tipo de inconveniente a la práctica del juego de bolos en los centros de enseñanza primaria de Boiro (p.e. maestro 1: yo inconveniente no, a no ser que... bueno, yo no le encuentro ningún inconveniente).

Respuesta/s

№. de menciones en las entrevistas
No le encuentro ningún inconveniente

No tenemos un espacio adecuado en el centro
5

4

2

Es una actividad individualista y poco dinámica 1

Tabla 6. Categoría 6. Maestros-inconvenientes de la práctica del juego de bolos en la escuela

El 50\% de las respuestas destacan como inconveniente la falta de espacio adecuado en los propios centros para realizar este tipo de actividades (p.e. maestro 7: inconveniente lo que 
decía antes, que no hay espacio físico en los centros para poder poner un campo de juego de bolos). Y ya en menor medida, se muestras respuestas (25\%) que indican que la peligrosidad que acompaña al juego de bolos es un inconveniente para realizar esta actividad en los colegios (p.e. maestro 7: tiene el inconveniente también de que es un juego peligroso) y respuestas $(12,5 \%)$ que subrayan la idea de que el juego de bolos es una actividad individualista y poco dinámica (p.e. maestro 8: es una actividad que no es muy dinámica de cara al trabajo con los niños). Comentábamos en la categoría 4 que la mayoría de los maestros no conocían la modalidad del juego de bolos de Boiro. Lo que practican en la escuela es una modalidad simple de bolos de derribo, o sea, lanzar rodando por el suelo una bola hacia unos bolos colocados verticalmente a una distancia determinada. Hacemos referencia a esto porque realmente el juego de bolos que se practica en Boiro (modalidad de pasabolo) es realmente peligroso si no se toman las medidas oportunas. Teniendo en cuenta que habría que adaptar el reglamento de juego y, sobre todo, el material empleado, los bolos no saldrían disparados como proyectiles ni se alcanzarían grandes distancias. Pero esto no implica que se tomen las debidas precauciones para evitar el impacto de un bolo, teniendo como referencia la práctica en un lugar con una pared alta de fondo para controlar el desplazamiento de los bolos,-separar debidamente las distintas zonas donde se efectúen los lanzamientos y tener la precaución de no lanzar cuando una persona se encuentre delante de los bolos, que aunque parezca evidente, a los niños y niñas les cuesta entender realmente el peligro de las cosas. Medidas como estas ayudan a controlar las condiciones de práctica de la actividad y reducir su peligrosidad cuando se realiza en un centro educativo; pero también estamos de acuerdo cuando se afirma que puede ser un juego individualista y poco dinámico, y que los centros no disponen de espacios adecuados. Estos inconvenientes se solucionarían adaptando las condiciones del juego a las posibilidades y recursos disponibles. Poco a poco los alumnos y alumnas tendrían la posibilidad de disfrutar de las condiciones reales del juego en campos de bolos habilitados en el municipio a medida que vayan creciendo.

En la categoría 7 (maestros-importancia de la escuela en la recuperación del juego de bolos) (ver tabla 7) el grupo más numeroso de respuestas $(87,5 \%)$ muestran la importancia de realizar la actividad bolística dentro de los propios centros y en horario escolar (p.e. maestro 4: en nuestras sesiones de Educación Física mostrarles una serie de juegos, entre ellos el de 
Artículo Original: La promoción de juegos populares y tradicionales en los centros de enseñanza de primaria del municipio de Boiro (A Coruña)

Vol. $n^{\circ}$ I, $n^{\circ} .1 ;$ p..53-74, Enero 2015. A Coruña. España ISSN 2386-8333 los bolos, para que ellos vayan habituándose a jugar o que le busquen las sensaciones positivas al juego y demás...).

\begin{tabular}{lc}
\hline \multicolumn{1}{c}{ Respuesta/s } & $\begin{array}{c}\text { №. de menciones en las } \\
\text { entrevistas }\end{array}$ \\
\hline Realizar la actividad en horario escolar & 7 \\
\hline $\begin{array}{l}\text { Realizar actividades en colaboración con las personas } \\
\text { mayores }\end{array}$ & 3 \\
\hline Realizar salidas a campos de bolos en activo & 2 \\
\hline Organizar actividades extraescolares & 2 \\
\hline $\begin{array}{l}\text { Realizar actividades en colaboración con diversas } \\
\text { entidades }\end{array}$ & 1 \\
\hline \multicolumn{1}{c}{ Tabla 7. Categoría 7. Maestros-importancia de la escuela en la recuperación del juego de bolos }
\end{tabular}

Destacan también las respuestas $(37,5 \%)$ que subrayan la importancia de realizar las actividades de bolos en los colegios contando con la colaboración de las familias de los niños y niñas y, principalmente, de abuelos (p.e. maestro 2: sería fundamental la colaboración de los mayores, que tengan experiencia y que seguramente pasaban las tardes de los domingos con la práctica de estos juegos). Un grupo menor de respuestas (25\%) muestran la necesidad de realizar salidas a campos de bolos en activo para observar sus características reales de juego y comprobar en directo como se juega a los bolos a través de exhibiciones de jugadores en activo (p.e. maestro 6: salir y llevar un día a los niños a un campo donde juegan ellos y que vean como se hace, y después motivarlos para que jueguen a esas cosas), y otras (25\%) que creen que sería adecuado exportar la actividad bolística fuera de los colegios, organizando actividades extraescolares (p.e. maestro 1: si no se realizan a nivel escolar, sino también a nivel extraescolar, yo creo que acabarán perdiéndose).

Y, por último, se muestran respuestas $(12,5 \%)$ que creen que es fundamental realizar la actividad en colaboración con diversas entidades deportivas, culturales y sociales (p.e. maestro 8: tener un mayor contacto a lo mejor con el ANPA, con asociaciones culturales..., para darle una continuidad fuera de lo que es el ámbito educativo).

Observamos como los maestros realmente entienden que si se pretende recuperar una actividad y promocionarla en diferentes ámbitos del municipio, la escuela juega un papel fundamental. Jugar a los bolos en los centros educativos implica que todos los niños y niñas van a conocer y practicar esa modalidad y, una vez que termine el horario escolar, los más 
Artículo Original: La promoción de juegos populares y tradicionales en los centros de enseñanza de primaria del municipio de Boiro (A Coruña)

Vol. $n^{\circ}$ I, no ${ }^{\circ} .1 ;$ p..53-74, Enero 2015. A Coruña. España ISSN 2386-8333 interesados y a los que realmente les guste el juego, continuarán practicándolo, como pasa con otros muchos juegos y deportes.

Los maestros deben ser consecuentes con sus palabras y colaborar directamente en la promoción del juego de bolos en el municipio de Boiro, como agentes directos en sus clases de Educación Física y también orientando, impulsando y motivando que su alumnado continúe con esta práctica fuera del ámbito educativo.

En la categoría 8 (maestros-los bolos fuera de la escuela) (ver tabla 8) la mayoría de las respuestas $(75 \%)$ afirman que lo que se aprende dentro de la escuela tiene una transferencia directa en la práctica extraescolar, realizando de forma autónoma las actividades que se aprendieron en la escuela y, entre ellas, podría estar el juego de bolos (p.e. maestro 6: todo a lo que juegan los niños en el colegio, cualquier juego que tu en el colegio lo metas en clase, en el recreo o en cualquier lado, como tenga un poco de continuidad y lo motives un poco, todos no, pero un porcentaje normal para estos casos, si que juegan fuera de la escuela).

Otras respuestas $(37,5 \%)$ indican que es necesario contar con la figura de un dinamizador fuera de la escuela para seguir con la práctica de los bolos que se inicie en los colegios (p.e. maestro 4: a lo mejor partiendo de alguna asociación que promueva un poco, el ayuntamiento también, o igual el ANPA poniendo un monitor fuera del horario escolar para que se haga cargo un poco de esto). En contraposición a esto, otro porcentaje similar de respuestas (37,5\%) afirman que es difícil que los niños jueguen fuera de la escuela (p.e. maestro 3: me parece difícil, habría que hacer una campaña bastante grande de información y concienciación para que enganche a los niños).

\begin{tabular}{lc}
\hline \multicolumn{1}{c}{ Respuesta/s } & $\begin{array}{c}\text { №. de menciones en las } \\
\text { entrevistas }\end{array}$ \\
\hline $\begin{array}{l}\text { Lo que se aprende en la escuela puede practicarse } \\
\text { fuera de ésta }\end{array}$ & 6 \\
\hline Es necesario un dinamizador fuera de la escuela & 3 \\
\hline Es difícil que los niños jueguen fuera de la escuela & 3 \\
\hline
\end{tabular}

Tabla 8. Categoría 8. Maestros-los bolos fuera de la escuela

Estamos de acuerdo con los maestros al afirmar que lo que se aprende en la escuela puede practicarse fuera, existiendo una transferencia real de conocimientos y aprendizajes del ámbito escolar fuera de éste (Woolfolk, 2010). De este modo se resalta aún más la importancia que tiene la práctica del juego de bolos en los centros educativos de la localidad, 
Artículo Original: La promoción de juegos populares y tradicionales en los centros de enseñanza de primaria del municipio de Boiro (A Coruña)

Vol. $n^{\circ}$ I, n ${ }^{\circ} .1$; p..53-74, Enero 2015. A Coruña. España ISSN 2386-8333 siendo el punto de partida, el inicio de una nueva generación de jugadores que serán los encargados de continuar y difundir esta tradición en un futuro próximo.

Pero al mismo tiempo es necesario formar una estructura estable que continúe con el trabajo que se empezó en los centros educativos. Aquí es donde adquiere una mayor importancia el Servicio Municipal de Deportes y el tejido asociativo de la localidad, encargados de recoger el relevo de los colegios y continuar con el trabajo fuera del ámbito escolar.

En la categoría 9 (maestros-causas del paulatino abandono del juego de bolos) (ver tabla 9) destacan las respuestas (50\%) que aluden a la aparición de las nuevas tecnologías la principal causa por la que el juego de bolos fue a menos en la localidad, afirmando que éstas fomentan el individualismo y el sedentarismo (p.e. maestro 3: la tecnología, tanta tecnología que hay ahora, tanta televisión... de muchas tecnologías).

\begin{tabular}{lc}
\hline \multicolumn{1}{c}{ Respuesta/s } & $\begin{array}{c}\text { №. de menciones en las } \\
\text { entrevistas }\end{array}$ \\
\hline Las nuevas tecnologías que fomentan el sedentarismo & 4 \\
\hline Irrupción de nuevos divertimientos & 3 \\
\hline Falta de tiempo de padres y madres & 3 \\
\hline Cambios en la sociedad, el progreso en si & 1 \\
\hline Aparición y auge del deporte del fútbol & 1 \\
\hline Migración del ámbito rural al núcleo urbano & 1 \\
\hline Tabla 9. Categoría 9. Maestros-causas del paulatino abandono del juego de bolos
\end{tabular}

En otro grupo de respuestas, ambas con el mismo porcentaje (37,5\%) se resalta la irrupción de nuevos divertimientos más modernos y novedosos como otra posible causa del decaimiento del juego (p.e. maestro 7: pero yo creo que una de esas causas es la oferta que hay de otro tipo de juegos que son más interesantes), y la falta de tiempo de los padres, que no juegan tanto con los hijos como antes, y por consiguiente a los niños y niñas sólo les llega la información de juegos y deportes actuales, los que más salen en los medios de comunicación, los más demandados, los más aclamados por la sociedad y, entre eses, no está el juego de 
bolos (p.e. maestro 4: pero la principal razón, en los domicilios y fuera del horario escolar, pienso que es la falta de tiempo de los padres y de las madres).

$\mathrm{Y}$ en menor medida aparecen tres grupos de respuestas, todas también con el mismo porcentaje $(12,5 \%)$ que indican que las causas por las que el juego de bolos fue a menos en su práctica son debidas a los cambios en la sociedad y al progreso en si (p.e. maestro 8: la sociedad cambió, y cambian los espacios, cambian mentalidades, cambia la manera de ver las cosas, cambia todo), a la aparición y auge del deporte del fútbol (p.e. maestro 7: el fútbol nos lo meten por todos lados y los niños no ven más que fútbol, prefieren jugar al fútbol) y al fenómeno de la migración de los ciudadanos del ámbito rural al núcleo urbano, perdiendo de este modo los hábitos y tradiciones que eran más propias de la vida en las aldeas (p.e. maestro 8: pasamos de una sociedad rural a una sociedad urbana).

En este apartado pensamos que los maestros muestran su opinión sobre el paulatino abandono del juego de bolos en Boiro desconociendo que fue un juego de hombres (no de niños). Las principales causas que nombran los docentes creemos que están más orientadas a la justificación del abandono progresivo de la práctica de actividad física y deporte por los niños y niñas y acentuado en etapas posteriores a la educación primaria (Palou, Ponseti, Gili, Borras y Vidal, 2005; Macarro, Romero y Torres, 2010) que al hecho concreto del abandono del juego de bolos en sí.

\section{Conclusiones}

Los juegos populares y tradicionales constituyen un apartado importante dentro del actual currículum de Educación Primaria para la Comunidad Autónoma de Galicia. Es por lo tanto de obligado cumplimiento para el/la maestro/a de Educación Física su promoción y difusión entre el alumnado, que verá enriquecido su bagaje cultural e histórico con actividades que desde siempre han estado muy cerca de su entorno familiar.

Debería incrementarse el nivel de práctica de estos juegos, normalmente vinculados a días festivos o de celebración en los centros. Los juegos populares y tradicionales necesitan dotarse de sentido, sentimiento y valor, aspectos que el docente debería perseguir a lo largo del curso (juego como medio) como parte única de su programación, buscando la importancia que realmente tienen este tipo de prácticas lúdicas. 
Artículo Original: La promoción de juegos populares y tradicionales en los centros de enseñanza de primaria del municipio de Boiro (A Coruña)

Vol. $n^{\circ}$ I, $n^{\circ}$. 1; p..53-74, Enero 2015. A Coruña. España ISSN 2386-8333

El juego de bolos, con una importante tradición en el municipio de Boiro, debería tratarse específicamente en los centros educativos, convirtiéndose en uno de los principiales agentes de promoción, difusión y recuperación de este juego popular y tradicional, en otros tiempos principal divertimiento de la población boirense (Rodríguez, Pazos y Palacios, 2014).

Cierto es que en esta promoción debería realizarse en colaboración con el Servicio Municipal de Deportes y otras entidades locales sin fin de lucro (clubs de bolos), resultando de esta forma un amplio proyecto del que formen parte también las familias para poner en contacto a diferentes generaciones en busca de un mismo fin.

Los cambios producidos en la sociedad en los últimos tiempos, las nuevas tecnologías o los nuevos divertimientos a los que los ciudadanos tuvieron acceso después de una época en la que la libertad estaba acotada, nos dejaron nuevos juegos, deportes y pasatiempos en detrimento de las prácticas lúdicas tradicionales transmitidas de generación en generación.

Las administraciones públicas tienen el deber de recuperar este tipo de juegos y poner en conexión al ciudadano con sus raíces y su historia, hecho que le permitirá enfocar el futuro con mayores y mejores posibilidades de ocio.

\section{Referencias bibliográficas}

1. Barroso, C. (2003). Las bases sociales de la ludopatía (Tesis de Doctorado). Facultad de Ciencias Políticas y Sociología. Universidad de Granada.

2. Bautista J.M. (2002). El juego como método didáctico: propuestas didácticas y organizativas. Granada: Adhara.

3. Canales, M. (2006). Metodologías de investigación social. Introducción a los oficios. Santiago de Chile: LOM.

4. Corbetta, P. (2007). Metodología y técnicas de investigación social. Madrid: McGrawHill.

5. Cortizas, A. (2001). Chirlosmirlos. Enciclopedia dos xogos populares. Vigo: Xerais.

6. Diario Oficial de Galicia (DOG) $\mathrm{n}^{\mathrm{o}}$ 132, de luns 9 de xullo de 2007. Decreto 130/2007, do 28 de xuño, polo que se establece o currículo da Educación Primaria na Comunidade Autónoma de Galicia (pp. 11.666-11.760). 
7. Diario Oficial de Galicia (DOG) $\mathrm{n}^{\mathrm{o}}$ 171, de martes 9 de setembro de 2014. Decreto 105/2014, de 4 de setembro, polo que se establece o currículo de Educación Primaria na Comunidade Autónoma de Galicia (pp. 37406-38087).

8. Expósito, J. (2006). El juego y deporte popular, tradicional y autóctono en la escuela. Los bolos huertanos y bolos cartageneros. Sevilla: Wanceulen.

9. García, E. y Alarcón, M.J. (2011). Influencia del juego infantil en el desarrollo y aprendizaje del niño y la niña. Revista Digital Educación Física y Deportes, 153, p.1. Recuperado de http://www.efdeportes.com/efd153/influencia-del-juego-infantil-en-eldesarrollo.htm

10. Lara, A.J. (2011). Transmission of social and educational values through sport. Journal of Sport and Health Research (2011), 3 (1), 5-6.

11. Lavega, P. (1994). El juego: teorías y características del mismo. El juego como actividad física organizada. Estrategias del juego. En Temario desarrollado de los contenidos específicos del área de Educación Física para el acceso al Cuerpo de Profesores de Enseñanza Secundaria, 41-68. Barcelona: Inde.

12. Lavega, P. (1996). Del joc a l'esport. Estudi de bitlles al Pla d'Urgell (Lleida) (Tesis de doctorado). Universidad de Barcelona: Departament de teoria i història de l'educació (Lleida).

13. Lavega, P. (2006). Los juegos y deportes tradicionales en Europa: entre la tradición y la modernidad. Revista APUNTS de Educación Física y Deportes, 85, 68-81.

14. Liñares, X.A. (2007). Ramón Martínez López. Santiago de Compostela: TesCtres Editores.

15. López, J.L. (2009). Los juegos tradicionales de lanzamiento y precisión en el aula de Educación Física. Una adaptación práctica. Revista digital Educación Física y Deportes, 136, p.1. http://www.efdeportes.com/efd136/los-juegos-tradicionales-delanzamiento.htm

16. Macarro, J., Romero, C. y Torres, J. (2010). Motivos de abandono de la práctica físico-deportiva de los estudiantes de Bachillerato de la provincia de Granada. Revista de Educación, 353, 495-519.

17. Melero, M. (2009). Aproximación científica a los juegos populares, tradicionales y autóctonos de España. Revista digital Educación Física y Deportes, 133, p.1. 
Recuperado de http://www.efdeportes.com/efd133/juegos-populares-tradicionales-yautoctonos.htm

18. Monroy, A. y Sáez, G. (2011). Teorías sobre el origen del juego. Revista Digital Educación Física y Deportes, 153, p.1. Recuperado de http://www.efdeportes.com/efd153/teorias-sobre-el-origen-del-juego.htm

19. Moreno, C. (1992). Juegos y deportes tradicionales en España. Madrid: Alianza.

20. Palou, P., Ponseti, X., Gili, M., Borras, P. y Vidal, J. (2005). Motivos para el inicio, mantenimiento y abandono de la práctica deportiva de los preadolescentes de la isla de Mallorca. Revista Apunts de Educación Física y Deportes, 81, 5-11.

21. Ponce, C. (2009). El juego como recurso educativo. Revista Digital de Innovación y Experiencias Educativas, 19, 1-9.

22. Renzi, G.M. (2009). Educación Física y su contribución al desarrollo integral de los niños en la primera infancia. Revista Iberoamericana de Educación, 50, 1-14. Recuperado de http://www.rieoei.org/deloslectores/2663Renzi.pdf.

23. Rodríguez, J.E. (2013). O estudo do xogo dos bolos en Boiro: aspectos históricos, estado actual de práctica e perspectivas de futuro. Tesis de doctorado. A Coruña: Universidad da Coruña

24. Rodríguez, J.E., Pazos, J.M. y Palacios, J. (2014). El juego de bolos de Boiro: la necesidad de reglamentación de una modalidad de pasabolo atípica en España. EmásF, Revista Digital de Educación Física, 28, 17-43. Recuperado de http://emasf.webcindario.com/El_juego_de_bolos_de_boiro.pdf

25. Romaní, A. (1979). Xogos infantiles de Galicia. Santiago: Follas Novas.

26. Romero, J.B. (2007). El juego deportivo y musical como transmisor de valores no violentos. Revista Wanceulen Educación Física Digital, 3, pp.1-10. Recuperado de http://rabida.uhu.es/dspace/bitstream/handle/10272/1707/ b1534966.pdf?sequence=1

27. Ruiz, G. (2011). Hábitos de práctica lúdica y deportiva en niños y niñas en edad escolar: un estudio transversal. EmásF, Revista Digital de Educación Física, 10, 115. Recuperado de http://emasf.webcindario.com/Habitos_de_practica_ludica_y_deportiva_en_escolares .pdf 
Artículo Original: La promoción de juegos populares y tradicionales en los centros de enseñanza de primaria del municipio de Boiro (A Coruña)

Vol. $n^{\circ}$ I, $n^{\circ} .1 ;$ p..53-74, Enero 2015. A Coruña. España ISSN 2386-8333

28. Ruiz, J.G. (2000). Estudios de los bolos en Asturias: aspectos histórico-culturales, modalidades, elementos y materiales de juego. Estado actual de su práctica. Tesis de doctorado. Granada: Universidad de Granada.

29. Tójar, J.C. (2006). Investigación cualitativa. Comprender y actuar. Madrid: La Muralla.

30. Vega, T. (1990). Dos enredos tradicionales de la comarca Navea-Bibei (Orense). Boletín de Estudios del Seminario "Fontán Sarmiento” de Hagiografría, Toponimia y Onomástica de Galicia, 11, Año 12, 60-65.

31. Veiga, F. (1998). Xogo popular galego e educación. Vixencia educativa e función de identificación cultural dos xogos e enredos tradicionais. Tesis de doctorado. Santiago de Compostela: Universidad de Santiago de Compostela.

32. Vigne, M. (2011). Las actividades tradicionales de ocio como reflejo de una sociedad. Revista Científica Digital Acción Motriz 7: 62-76. http://www.accionmotriz.com/revistas/7/7_6.pdf.

33. Woolfolk, A. (2010). Psicología educativa. México: Pearson Educación. 